\title{
Nonlinear Schrödinger equations from prequantum classical statistical field theory
}

\author{
Andrei Khrennikov \\ International Center for Mathematical Modeling \\ in Physics and Cognitive Sciences \\ University of Växjö, Sweden \\ Email:Andrei.Khrennikov@msi.vxu.se
}

\begin{abstract}
We derive some important features of the standard quantum mechanics from a certain classical-like model - prequantum classical statistical field theory, PCSFT. In this approach correspondence between classical and quantum quantities is established through asymptotic expansions. PCSFT induces not only linear Schrödinger's equation, but also its nonlinear generalizations. This coupling with "nonlinear wave mechanics" is used to evaluate the small parameter of PCSFT.

Keywords: prequantum classical statistical field theory, asymptotic expansions, nonlinear wave mechanics
\end{abstract}

\section{Introduction}

We demonstrated in [1] that averages of quantum observables can be considered as approximations of averages of classical variables on Hilbert phase space, see similarities but also differences with the schemes of [2]-[4] (see also [5]-[12] on various attempts to go beyond quantum mechanics). By choosing a special representation of the infinite dimensional configuration space, namely as the $L_{2}\left(\mathbf{R}^{3}\right)$-space, we obtain the following representation of the phase space:

$$
\Omega=L_{2}\left(\mathbf{R}^{3}\right) \times L_{2}\left(\mathbf{R}^{3}\right) .
$$


In such a representation points of the prequantum phase space can be interpreted as classical (vector) fields, $\psi(x)=(q(x), p(x))$. Therefore we called our model prequantum classical statistical field theory, PCSFT.

The correspondence between PCSFT and QM is asymptotic: we expand classical ("prequantum") averages with respect to a small parameter $\alpha$ - the dispersion of vacuum fluctuations. The main term in this expansion is given by the quantum average. We call our approach asymptotic dequantization. We emphasize that we develop the classical (statistical) theory which does not reproduce exactly $Q M$. There is only an asymptotic coupling between classical and quantum models. To come with concrete experimental predictions, we should evaluate the small parameter $\alpha$ of the model. We do this in the present note by exploring the relation between PCSFT and theory of nonlinear Schrödinger's equation - "nonlinear wave mechanics," [13]-[16].

We point out that PCSFT induces not only conventional linear Schrödinger's equation, but also so called nonlinear Schrödinger's equations, cf. [13]-[16]. In PCSFT these nonlinear Schrödinger equations appear very naturally as infinite dimensional Hamilton equations. Hence the PCSFT-approach to QM could be considered as additional justification of nonlinearity of dynamics on prequantum scales. On the other hand, since the small parameter $\alpha$ of PCSFT can be identified with the coupling constant (denoted by $b$ in [13], [14], [16]) in nonlinear Schrödinger equations, we can apply experimental estimates [14] for this coupling constant to evaluate the $\alpha$, see section 7 .

\section{Asymptotic statistical correspondence between classical and quantum models}

We define "classical statistical models" in the following way, see [1] for more detail: a) physical states $\omega$ are represented by points of some set $\Omega$ (state space); b) physical variables are represented by functions $f: \Omega \rightarrow \mathbf{R}$ belonging to some functional space $V(\Omega)$; c) statistical states are represented by probability measures on $\Omega$ belonging to some class $S(\Omega)$; d) the average of a physical variable (which is represented by a function $f \in V(\Omega)$ ) with respect to a statistical state (which is represented by a probability measure $\rho \in S(\Omega)$ ) is given by

$$
<f>_{\rho} \equiv \int_{\Omega} f(\psi) d \rho(\psi)
$$


A classical statistical model is a pair $M=(S, V)$. We recall that classical statistical mechanics on the phase space $\Omega_{2 n}=\mathbf{R}^{n} \times \mathbf{R}^{n}$ gives an example of a classical statistical model. But we shall not be interested in this example in our further considerations. We shall develop a classical statistical model with infinite-dimensional phasespace.

The conventional quantum statistical model with the complex Hilbert state space $\Omega_{c}$ is described in the following way (see Dirac-von Neumann [17], [18] for the conventional complex model): a) physical observables are represented by operators $A: \Omega_{c} \rightarrow \Omega_{c}$ belonging to the class of continuous self-adjoint operators $\mathcal{L}_{s} \equiv \mathcal{L}_{s}\left(\Omega_{c}\right)$; b) statistical states are represented by von Neumann density operators, see [18] (the class of such operators is denoted by $\mathcal{D} \equiv \mathcal{D}\left(\Omega_{c}\right)$ ); d) the average of a physical observable (which is represented by the operator $A \in \mathcal{L}_{s}\left(\Omega_{c}\right)$ ) with respect to a statistical state (which is represented by the density operator $D \in \mathcal{D}\left(\Omega_{c}\right)$ ) is given by von Neumann's formula [18]:

$$
<A>_{D} \equiv \operatorname{Tr} \mathrm{DA}
$$

The quantum statistical model is the pair $N_{\text {quant }}=\left(\mathcal{D}, \mathcal{L}_{s}\right)$.

We are looking for a classical statistical model $M=(S, V)$ which will give "dequantization" of the quantum model $N_{\text {quant }}=\left(\mathcal{D}, \mathcal{L}_{s}\right)$. Here the meaning of "dequantization" should be specified. In fact, all "NO-GO" theorems (e.g., von Neumann, Kochen-Specker, Bell,...) can be interpreted as theorems about impossibility of various dequantization procedures. Therefore we should define the procedure of dequantization in such a way that there will be no contradiction with known "NO-GO" theorems, but our dequantization procedure still will be natural from the physical viewpoint. We define (asymptotic) dequantization as a family $M^{\alpha}=\left(S^{\alpha}, V\right)$ of classical statistical models depending on a small parameter $\alpha \geq 0$. There should exist maps $T: S^{\alpha} \rightarrow \mathcal{D}$ and $T: V \rightarrow \mathcal{L}_{s}$ such that: a) both maps are surjections (so all quantum states and observables can be represented as images of classical statistical states and variables, respectively); b) the map $T: V \rightarrow \mathcal{L}_{s}$ is $\mathbf{R}$-linear (we recall that we consider real-valued classical physical variables); c) classical and quantum averages are coupled through the following asymptotic equality:

$$
<f>_{\rho}=\frac{\alpha}{2}<T(f)>_{T(\rho)}+o(\alpha), \quad \alpha \rightarrow 0
$$

(here $<T(f)>_{T(\rho)}$ is the quantum average). This equality can be interpreted in the following way. Let $f(\psi)$ be a classical physical vari- 
able (describing properties of microsystems - classical fields having very small magnitude $\alpha$ ). We define its amplification by: $f_{\alpha}(\psi)=\frac{1}{\alpha} f(\psi)$. If we interpret $\alpha$ as the intensity of vacuum fluctuations, then $f_{\alpha}(\psi)$ is the relative intensity of $f(\psi)$ with respect to vacuum fluctuations. By dividing both sides of the equation (4) by $\alpha$ we obtain:

$$
<f_{\alpha}>_{\rho}=\frac{1}{2}<T(f)>_{T(\rho)}+o(1), \alpha \rightarrow 0,
$$

Hence: The quantum term gives the main contribution into the relative intensity with respect to vacuum fluctuations. The crucial difference from dequantizations considered in known "NO-GO" theorems (e.g., von Neumann, Kochen-Specker, Bell) is that in our case classical and quantum averages are equal only asymptotically.

\section{Prequantum classical statistical model with the infinite dimensional phase space}

We choose the phase space $\Omega=Q \times P$, where $Q=P=H$ and $H$ is the infinite-dimensional real (separable) Hilbert space. We consider $\Omega$ as the real Hilbert space with the scalar product $\left(\psi_{1}, \psi_{2}\right)=$ $\left(q_{1}, q_{2}\right)+\left(p_{1}, p_{2}\right)$. We denote by $J$ the symplectic operator on $\Omega: J=$ $\left(\begin{array}{ll}0 & 1 \\ -1 & 0\end{array}\right)$. Let us consider the class $\mathcal{L}_{\text {symp }}(\Omega)$ of bounded $\mathbf{R}$-linear operators $A: \Omega \rightarrow \Omega$ which commute with the symplectic operator:

$$
A J=J A
$$

This is a subalgebra of the algebra of bounded linear operators $\mathcal{L}(\Omega)$. We also consider the space of $\mathcal{L}_{\text {symp, }}(\Omega)$ consisting of self-adjoint operators.

By using the operator $J$ we can introduce on the phase space $\Omega$ the complex structure. Here $J$ is realized as $-i$. We denote $\Omega$ endowed with this complex structure by $\Omega_{c}: \Omega_{c} \equiv Q \oplus i P$. We shall use it later. At the moment consider $\Omega$ as a real linear space and consider its complexification $\Omega^{\mathbf{C}}=\Omega \oplus i \Omega$.

Let us consider the functional space $\mathcal{V}_{\text {symp }}(\Omega)$ consisting of functions $f: \Omega \rightarrow \mathbf{R}$ such that: a) the state of vacuum is preserved : $f(0)=0$; b) $f$ is $J$-invariant: $f(J \psi)=f(\psi)$; c) $f$ can be extended to the analytic function $f: \Omega^{\mathbf{C}} \rightarrow \mathbf{C}$ having the exponential growth: $|f(\psi)| \leq c_{f} e^{r_{f}}\|\psi\|$ for some $c_{f}, r_{f} \geq 0$ and for all $\psi \in \Omega^{\mathbf{C}}$. We remark 
that the possibility to extend a function $f$ analytically onto $\Omega^{\mathbf{C}}$ and the exponential estimate on $\Omega^{\mathrm{C}}$ plays the important role in the asymptotic expansion of integrals over the infinite dimensional phase space. ${ }^{1}$ But this is purely mathematical condition which could be in principle weakened.

The following elemntary mathematical result plays the fundamental role in establishing classical $\rightarrow$ quantum correspondence: Let $f$ be a smooth $J$-invariant function. Then $f^{\prime \prime}(0) \in \mathcal{L}_{\text {symp,s }}(\Omega)$. In particular, a quadratic form is $J$-invariant iff it is determined by an operator belonging to $\mathcal{L}_{\text {symp,s }}(\Omega)$.

We consider the space statistical states $S_{\text {symp }}^{\alpha}(\Omega)$ consisting of measures $\rho$ on $\Omega$ such that: a) $\rho$ is symmetric (in particular, its mean value equals to zero); b) the dispersion of $\rho$ is equal to $\alpha$ :

$$
\sigma^{2}(\rho)=\int_{\Omega}\|\psi\|^{2} d \rho(\psi)=\alpha, \alpha \rightarrow 0 ;
$$

c) $\rho$ is $J$-invariant; d) for any $r>0$ the exponential function $e^{r\|\psi\|}$ is integrable. The latter condition is a purely mathematical one and it could be weakened. Such measures describe small statistical fluctuations of the vacuum field.

The following trivial mathematical result plays the fundamental role in establishing classical $\rightarrow$ quantum correspondence: Let a measure $\rho$ be J-invariant. Then its covariation operator $B=\operatorname{cov} \rho \in$ $\mathcal{L}_{\text {symp,s }}(\Omega)$. Here $\left(B y_{1}, y_{2}\right)=\int\left(y_{1}, \psi\right)\left(y_{2}, \psi\right) d \rho(\psi)$.

We now consider the complex realization $\Omega_{c}$ of the phase space and the corresponding complex scalar product $\langle\cdot, \cdot\rangle$. We remark that the class of operators $\mathcal{L}_{\text {symp }}(\Omega)$ is mapped onto the class of $\mathbf{C}$-linear operators $\mathcal{L}\left(\Omega_{c}\right)$.

We also define for any measure its complex covariation operator $B^{c}=\operatorname{cov}^{\mathrm{c}} \rho$ by $<B^{c} y_{1}, y_{2}>=\int<y_{1}, \psi><\psi, y_{2}>d \rho(\psi)$. We remark that for a $J$-invariant measure $\rho$ its complex and real covariation operators are related as $B^{c}=2 B$.

As in the real case [1], we can prove that for any operator $A \in$ $\mathcal{L}_{\text {symp,s }}(\Omega)$ :

$$
\int_{\Omega}<A \psi, \psi>d \rho(\psi)=\operatorname{Tr} \operatorname{cov}^{\mathrm{c}} \rho \mathrm{A} .
$$

We pay attention that the trace is considered with respect to the complex inner product. We remark that this formula has been already

\footnotetext{
${ }^{1}$ To get a mathematically rigor formulation, conditions in [1] should be reformulated in the similar way.
} 
used by Bach [19] for representing quantum averages. The crucial difference from Bach's approach is that we consider arbitrary functionals of $\psi$ and we shall obtain an asymptotic relation between classical and quantum averages.

We consider now the one parameter family of classical statistical models:

$$
M^{\alpha}=\left(S_{\text {symp }}^{\alpha}(\Omega), \mathcal{V}_{\text {symp }}(\Omega)\right), \alpha \geq 0
$$

Theorem 1. Let $f \in \mathcal{V}_{\text {symp }}(\Omega)$ and let $\rho \in S_{\text {symp }}^{\alpha}(\Omega)$. Then the following asymptotic equality holds:

$$
<f>_{\rho}=\frac{\alpha}{2} \operatorname{Tr} \mathrm{D}^{\mathrm{c}} \mathrm{f}^{\prime \prime}(0)+\mathrm{o}(\alpha), \alpha \rightarrow 0,
$$

where the operator $D^{c}=\operatorname{cov}^{\mathrm{c}} \rho_{\text {scal }}$ and $\rho_{\text {scal }}$ is the $\sqrt{\alpha}$-scaling of the measure $\rho$. Here o $(\alpha)=\alpha^{2} R(\alpha, f, \rho)$, where $|R(\alpha, f, \rho)| \leq c_{f} \int_{\Omega} e^{r_{f}\|\Psi\|} d \rho_{\text {scal }}(\Psi)$.

Here and everywhere below we use the symbols $\psi$ and $\Psi$ to denote "prequantum field" and its scaling, respectively:

$$
\psi=\sqrt{\alpha} \Psi .
$$

We see that the classical average (computed in the model $M^{\alpha}=$ $\left(S_{\text {symp }}^{\alpha}(\Omega), \mathcal{V}_{\text {symp }}(\Omega)\right)$ by using the measure-theoretic approach) is coupled through (9) to the quantum average (computed in the model $N_{\text {quant }}=\left(\mathcal{D}\left(\Omega_{c}\right), \mathcal{L}_{\mathrm{s}}\left(\Omega_{c}\right)\right)$ by the von Neumann trace-formula).

The equality (9) can be used as the motivation for defining the following classical $\rightarrow$ quantum map $T$ from the classical statistical model $M^{\alpha}=\left(S_{G, \text { symp }}^{\alpha}, \mathcal{V}_{\text {symp }}\right)$ onto the quantum statistical model $N_{\text {quant }}=\left(\mathcal{D}, \mathcal{L}_{\mathrm{S}}\right)$ :

$$
T: S_{\text {symp }}^{\alpha}(\Omega) \rightarrow \mathcal{D}\left(\Omega_{c}\right), \quad D^{c}=T(\rho)=\operatorname{cov}^{\mathrm{c}} \rho_{\text {scal }}
$$

(the measure $\rho$ is represented by the density matrix $D^{c}$ which is equal to the complex covariation operator of its $\sqrt{\alpha}$-scaling );

$$
T: \mathcal{V}_{\text {symp }}(\Omega) \rightarrow \mathcal{L}_{\mathrm{s}}\left(\Omega_{c}\right), \quad A_{\text {quant }}=T(f)=f^{\prime \prime}(0) .
$$

Our previous considerations can be presented as

Theorem 2. The one parametric family of classical statistical models $M^{\alpha}=\left(S_{\mathrm{symp}}^{\alpha}(\Omega), \mathcal{V}_{\mathrm{symp}}(\Omega)\right)$ provides asymptotic dequantization of the quantum model $N_{\text {quant }}=\left(\mathcal{D}\left(\Omega_{c}\right), \mathcal{L}_{\mathrm{S}}\left(\Omega_{c}\right)\right)$ through the pair of maps (11) and (12). The classical and quantum averages are coupled by the asymptotic equality (5). 


\section{Infinite dimension of physical space}

This is a good place to discuss the model of physical space in PCSFT. Here the real physical space is Hilbert space. If we choose the realization $H=L_{2}\left(\mathbf{R}^{3}\right)$, then we obtain the realization of $H$ as the space of classical fields on $\mathbf{R}^{3}$. So the conventional space $\mathbf{R}^{3}$ appears only through this special representation of the Hilbert configuration space. Dynamics in $\mathbf{R}^{3}$ in just a shadow of dynamics in the space of fields. However, we can choose other representations of the Hilbert configuration space. In this way we shall obtain classical fields defined on other "physical spaces."

We remark that at the first sight the situation with development of PCSFT is somewhat reminiscent of the one confronted by Scrödinger in his introduction of his wave equation, which "maps" waves in the configuration space. However, even though he had, just as did Einstein, major reservations concerning quantum mechanics as the ultimate theory of quantum phenomena, Scrödinger never went so far as to see any space other than $\mathbf{R}^{3}$ as real. ${ }^{2}$

On the other hand, string theory does introduce spaces of higher dimensions, although not of infinite dimensions. This approach was one of inspirations for our radical viewpoint to physical space. One could speculate that on scales of quantum gravity and string theory space became infinite dimensional, just as those theories the space has the (finite) dimension higher than three. ${ }^{3}$

\section{$5 \quad$ Hamilton-Schrödinger dynamics}

States of systems with the infinite number of degrees of freedom classical fields - are represented by points $\psi=(q, p) \in \Omega$; evolution of

\footnotetext{
${ }^{2}$ We point out L. De Broglie in his theory of double solution emphasized the fundamental role of physical space $\mathbf{R}^{3}$. Such a viewpoint also was common for adherents of Bohmian mechanics (in any case for D. Bohm and J. Bell). However, recently B. Hiley started to consider the momentum representation of Bohmian mechanics [20] and it seems that in Hiley's approach to Bohmian mechanics the position representation does not play an exceptional role.

${ }^{3}$ In our approach quantum theory is not the ultimate theory. It has its boundaries of applications. Therefore there are no reasons to expect that "quantum gravity" should exist at all. Thus it would be better to speak not about scales of quantum gravity and string theory, but simply about the Planck scale for length and time.
} 
a state is described by the Hamiltonian equations:

$$
\dot{q}=\frac{\partial \mathcal{H}}{\partial p}, \dot{p}=-\frac{\partial \mathcal{H}}{\partial q} .
$$

First we consider a quadratic Hamilton function: $\mathcal{H}(q, p)=\frac{1}{2}(\mathbf{H} \psi, \psi)$, where $\mathbf{H}: \Omega \rightarrow \Omega$ is an arbitrary symmetric (bounded) operator. In this special case the Hamiltonian equations have the form: $\dot{q}=\mathbf{H}_{21} q+$ $\mathbf{H}_{22} p, \quad \dot{p}=-\left(\mathbf{H}_{11} q+\mathbf{H}_{12} p\right)$, or $\dot{\psi}=\left(\begin{array}{c}\dot{q} \\ \dot{p}\end{array}\right)=J \mathbf{H} \psi$. Thus quadratic Hamilton functions induce linear Hamilton equations. We get $\psi(t)=$ $U_{t} \psi$, where $U_{t}=e^{J \mathbf{H} t}$. The map $U_{t} \psi$ is a linear Hamiltonian flow on the phase space $\Omega$.

Let us now consider an operator $\mathbf{H} \in \mathcal{L}_{\text {symp }, \mathrm{s}}(\Omega): \mathbf{H}=\left(\begin{array}{ll}R & T \\ -T & R\end{array}\right)$. This operator defines the quadratic Hamilton function $\mathcal{H}(q, p)=$ $\frac{1}{2}[(R p, p)+2(T p, q)+(R q, q)]$, where $R^{*}=R, T^{*}=-T$. Corresponding Hamiltonian equations have the form $\dot{q}=R p-T q, \dot{p}=-(R q+T p)$. We pay attention that for a $J$-invariant Hamilton function, the Hamiltonian flow $U_{t} \in \mathcal{L}_{\text {symp }}(\Omega)$. By considering the complex structure on the infinite-dimensional phase space $\Omega$ we write the latter Hamiltonian equations in the form of the Schödinger equation on $\Omega_{c}$ :

$$
i \frac{d \psi}{d t}=\mathbf{H} \psi
$$

its solution has the following complex representation: $\psi(t)=U_{t} \psi, U_{t}=$ $e^{-i \mathbf{H} t}$. We consider the Planck system of units in that $h=1$. This is the complex representation of flows corresponding to quadratic $J$-invariant Hamilton functions.

However, in our approach there are no reasons to restrict considerations by quadratic ( $J$-invariant) Hamilton functions. We can take any function $\mathcal{H} \in C^{1}(\Omega)$ as the Hamilton function. It induces the Hamilton-Schrödinger dynamics:

$$
i \frac{d \psi}{d t}=\mathcal{H}^{\prime}(\psi)
$$

We pay attention that the Hamilton-Schrödinger equation can be considered for any Hamilton function which is one time continuously Frechet-differentiable on the infinite dimensional phase space. However, we obtained the asymptotic expansion of classical average (on the infinite dimensional phase space) only for analytic physical variables. 
Our approach could be generalized for functions of the class $C^{2}$. But if a function does not have the second Frechet-derivative, then we are not able to apply technique based on the Taylor expansion and obtain quantum effects (which give the contribution of the second order in the Taylor formula), cf. problems which will be discussed in section 6 .

\section{Scaling of prequantum variables}

Considerations of section 3, as well as everywhere in [1], were based on scaling of probability measures representing classical statistical states. Since there is the natural duality between measures and functions, it is possible to consider scaling of classical variables, instead of scaling of measures. It is important to consider scaling of variables, because the basic equation of quantum mechanics is Schrödinger's equation (which is an image of the classical Hamiltonian equation). For any function $f: \Omega \rightarrow \mathbf{R}$ we set

$$
f_{Q}(\Psi)=\frac{1}{\alpha} f(\sqrt{\alpha} \Psi) .
$$

The $f_{Q}$ is the result of the transition from the prequantum system of the $\psi$-field coordinates to the quantum system of the $\Psi$-field coordinates and $1 / \alpha$-amplification of the classical variable $f$. Such a renormalization of $f$ can be justified in two ways: a) through statistical averages, b) through the Hamiltonian-Schrödinger equations.

\subsection{Statistical origin of the renormalization}

The asymptotic representation of the classical average can be written as

$$
\begin{aligned}
<f / \alpha>_{\rho} & =\int_{\Omega} \frac{f(\sqrt{\alpha} \Psi)}{\alpha} d \rho_{\text {scal }}(\Psi) \equiv \int_{\Omega} f_{Q}(\Psi) d \rho_{\text {scal }}(\Psi) \\
& =\frac{1}{2} \operatorname{Tr} \operatorname{cov}^{\mathrm{c}} \rho_{\text {scal }} \mathrm{f}^{\prime \prime}(0)+\mathrm{o}(1), \alpha \rightarrow 0
\end{aligned}
$$

We remark that $\frac{\partial^{2} f}{\partial \psi^{2}}(0)=\frac{\partial^{2} f_{Q}}{\partial \Psi^{2}}(0)$. Thus we have: $<f_{Q}>_{\rho_{\text {scal }}}=$ $\frac{1}{2} \operatorname{Tr} \operatorname{cov}^{\mathrm{c}} \rho_{\text {scal }} \mathrm{f}_{\mathrm{Q}}^{\prime \prime}(0)+\mathrm{o}(1), \alpha \rightarrow 0$. Therefore we can consider a new classical model in that statistical states are given by measures $\mu \in$ $S_{\text {symp }}(\Omega)$ (having dispersion 1 ) and physical variables by functions $g \in \mathcal{V}_{\text {symp }}(\Omega)$ (we note that any function $g$ can be considered as scaling for some $f$ belonging the same functional space: $g(\Psi) \equiv f_{Q}(\Psi)=$ 
$f(\sqrt{\alpha} \Psi) / \alpha) .{ }^{4}$ We consider the classical statistical model $M=\left(S_{\text {symp }}(\Omega), \mathcal{V}_{\text {symp }}(\Omega)\right)$ and the map $T: M \rightarrow N_{\text {quant }}$, given by:

$$
\begin{aligned}
& T(\mu)=\operatorname{cov}^{c} \mu \\
& T(g)=g^{\prime \prime}(0)
\end{aligned}
$$

This map provides dequantization of $\mathbf{N}$ and the following asymptotic equality holds:

$$
<f_{Q}>_{\mu}=\frac{1}{2}<T\left(f_{Q}\right)>_{T(\mu)}+o(1), \alpha \rightarrow 0 .
$$

\subsection{Dynamical origin of renormalization}

Let us consider a Hamilton function $\mathcal{H}(\psi)$. The corresponding Hamilton equation is given by (14). We now change the system of coordinates: $\psi=\sqrt{\alpha} \Psi$ and obtain the Hamilton equation in the $\Psi$ coordinates:

$$
i \dot{\Psi}=\mathcal{H}_{Q}^{\prime}(\Psi)
$$

If $\mathcal{H}(\psi)$ is a quadratic (J-invariant) function, then $\mathcal{H}(\psi) \equiv \mathcal{H}_{Q}(\Psi)$ and we obtain the conventional Schrödinger equation. Thus we came again to the same procedure of renormalization $\mathcal{H} \rightarrow \mathcal{H}_{Q}$ of classical physical variables. If $\mathcal{H}$ is not quadratic, then the Schrödinger-Hamilton equation (19) gives, in particular, so called nonlinear Schrödinger equations, see, e.g., [13]-[16]. For example, let $\mathcal{H}(\psi)=\frac{1}{2}(\hat{H} \psi, \psi)+\frac{1}{4} \mathcal{H}_{4}(\psi, \psi, \psi, \psi)$, where $\mathcal{H}_{4}$ is a $J$-invariant form of degree four. Then

$$
\mathcal{H}_{Q}(\Psi)=\frac{1}{2}(\hat{H} \Psi, \Psi)+\frac{\alpha}{4} \mathcal{H}_{4}(\Psi, \Psi, \Psi, \Psi) .
$$

Hence, the corresponding Schrödinger-Hamilton equation has the form:

$$
i \dot{\Psi}=\hat{H} \Psi+\alpha \mathcal{H}_{4}^{\prime}(\Psi, \Psi, \Psi) .
$$

For example, let $\Omega=L_{2}^{c}\left(\mathbf{R}^{3}\right)$ and let

$$
\mathcal{H}_{4}(\Psi, \Psi, \Psi, \Psi)=\int_{\mathbf{R}^{12}} K_{4}\left(x_{1}, x_{2}, x_{3}, x_{4}\right) \Psi\left(x_{1}\right) \Psi\left(x_{2}\right) \overline{\Psi\left(x_{3}\right)} \overline{\Psi\left(x_{4}\right)} d x_{1}^{3} \ldots d x_{4}^{3} .
$$

\footnotetext{
${ }^{4}$ In other words this functional space is invariant with respect to the scaling map: $f \rightarrow f_{Q}$.
} 
By choosing the kernel $k\left(x_{1}, x_{2}, x_{3}, x_{4}\right)=\delta\left(x_{1}-x_{2}\right) \delta\left(x_{2}-x_{3}\right) \delta\left(x_{3}-x_{4}\right)$, we obtain the Hamilton function with nonquadratic term: $\mathcal{H}_{4}(\Psi, \Psi, \Psi, \Psi)=$ $\int_{\mathbf{R}^{3}}|\Psi(x)|^{4} d x$, and the well known nonlinear Schrödinger's equation

$$
i \frac{\partial \Psi}{\partial t}(t, x)=-\frac{1}{2} \Delta \Psi(t, x)+V(x) \Psi(t, x)+\alpha|\Psi(t, x)|^{2} \Psi(t, x) .
$$

However, PCSFT induces the class of Schrödinger-Hamilton equations which is essentially larger than the conventional class of nonlinear Schrödinger's equations with polynomial nonlinearities. For example, let us choose $\mathcal{H}_{4}(\Psi, \Psi, \Psi, \Psi)=\frac{1}{4}\left(\Gamma_{1} \Psi, \Psi\right)\left(\Gamma_{2} \Psi, \Psi\right)$, where $\Gamma_{j}: \Omega \rightarrow \Omega$ are $J$-invariant linear operators. We obtain the Schrödinger-Hamilton equation:

$$
i \frac{\partial \Psi}{\partial t}=\hat{H} \Psi+\frac{\alpha}{2}\left[\left(\Gamma_{1} \Psi, \Psi\right) \Gamma_{2} \Psi+\left(\Gamma_{2} \Psi, \Psi\right) \Gamma_{1} \Psi\right]
$$

On the other hand, in conventional nonlinear wave mechanics there were considered equations corresponding to Hamilton functions $\mathcal{H}(\psi)$ which are not analytic (and even not twice differentiable). The most important example are log-nonlinearities, see [13]-[16]. We shall come back to this question in section 7 .

\section{$7 \quad$ An estimation of the small parameter $\alpha$ through theory of nonlinear Schrödinger equations}

The derivation of nonlinear Schrödinger equations in the standard Hamiltonian formalism (but on the infinite dimensional phase space) is an important consequence of PCSFT. Nonlinear Schrödinger's equations were considered in many papers, see, e.g., [13]-[16]. And the problem of experimental verification was already studied [14], [16]. In PCSFT the parameter $\alpha$ can also be interpreted as the coupling constant for nonlinear perturbations of the Schrödinger equation. Hence, the upper bound for such a constant that was obtained in theory of nonlinear Schrödinger equations [14], [16] is also valid for the small parameter $\alpha$ of PCSFT.

The main problem in applications of results obtained in [13]-[16] for evaluating the small parameter $\alpha$ of PCSFT is that research in the conventional nonlinear wave mechanics was mainly concentrated on the 
equation with log-nonlinearity. I know only one paper on experimental estimation of the coupling constant for nonlog-nonlinearities, see [14] (Weinberg), but even in this paper finally there was considered the log-case. We recall that by reasons of locality the following equation plays the fundamental role in conventional nonlinear mechanics, see [15]:

$$
i \frac{\partial \Psi}{\partial t}=-\frac{1}{2} \Delta \Psi+V \Psi+b \ln |\Psi|^{2} \Psi
$$

There was obtained the experimental estimate of the coupling constant $b$, see [14], [16]:

$$
|b| \leq 10^{-15} \mathrm{eV} .
$$

We pay attention that (23) is the Hamilton equation on $\Omega$ with the Hamilton function

$$
\mathcal{H}_{Q}(\Psi)=\frac{1}{2} \int_{\mathbf{R}^{3}}\left[\frac{|\nabla \Psi(x)|^{2}}{2}+b|\Psi(x)|^{2}\left(\ln |\Psi(x)|^{2}-1\right)\right] d^{3} x .
$$

The main problem is that this function is not of the $C^{2}$-class on the phase space $\Omega=L_{2}\left(\mathbf{R}^{3}\right) \times L_{2}\left(\mathbf{R}^{3}\right)$. Therefore we could not use the Taylor expansion and apply directly our scheme of asymptotic dequantization. Nevertheless, let us try to proceed.

We now should be careful with dimensions of quantities under consideration. By our interpretation [1] of the background random field $\psi(x)$, the $|\psi(x)|^{2}$ has the dimension of the density of energy: $|\psi(x)|^{2} \sim E / L^{3}$. Since for the conventional $\Psi$-function, the $|\Psi(x)|^{2}$ has the dimension $\sim 1 / L^{3}$, we have that $\alpha$ has the dimension of energy. Let us write a prequantum Hamilton function inducing the log-nonlinearity by taking into account dimensions of quantities. We start by repeating previous considerations for quantities with physical dimensions. First we consider dynamics of the background $\psi$ field. The Hamilton equation should be, in fact, written in the form $i \tau \frac{\partial \psi}{\partial t}=\mathcal{H}^{\prime}(\psi)$, where $\tau$ has the dimension of time. We choose $\tau=t_{P}$ the Planck time. By setting now $\mathcal{H}_{Q}(\Psi)=\left(\frac{E_{P}}{\alpha}\right) \mathcal{H}(\sqrt{\alpha} \Psi)$, we can write our equation in the $\Psi$-system of coordinates

$$
i h \frac{\partial \Psi}{\partial t}=\mathcal{H}_{Q}^{\prime}(\Psi)
$$

For example, we know that for a nonrelativistic quantum particle in the QM-approximation:

$$
\mathcal{H}_{Q}(\Psi)=\int_{\mathbf{R}^{3}}\left[\frac{h^{2}}{2 m}|\nabla \Psi(x)|^{2}+V(x)|\Psi(x)|^{2}\right] d^{3} x
$$


Therefore the corresponding prequantum Hamilton function:

$$
\mathcal{H}(\psi)=\int_{\mathbf{R}^{3}}\left[\frac{h^{2}}{2 m E_{P}}|\nabla \psi(x)|^{2}+\frac{V(x)}{E_{P}}|\psi(x)|^{2}\right] d^{3} x .
$$

Here we have used the relation: $\mathcal{H}(\psi)=\left(\frac{\alpha}{E_{P}}\right) \mathcal{H}_{Q}\left(\frac{\psi}{\sqrt{\alpha}}\right)$. In the real physical dimensions the log-term of $\mathcal{H}_{Q}(\Psi)$, see (25), should be written as

$$
U_{Q}(\Psi)=b \int_{\mathbf{R}^{3}}|\Psi(x)|^{2}\left(\ln a^{3}|\Psi(x)|^{2}-1\right) d^{3} x,
$$

where $a \sim L$ (since $\left.|\Psi(x)|^{2} \sim 1 / L^{3}\right)$. Its prequantum counterpart

$$
U(\psi)=\left(\frac{\alpha}{E_{P}}\right)\left(\frac{b}{\alpha}\right) \int_{\mathbf{R}^{3}}|\psi(x)|^{2}\left(\ln \frac{a^{3}}{\alpha}|\psi(x)|^{2}-1\right) d^{3} x .
$$

It is natural to identify the energy $b$-scale corresponding to the nonlinear perturbation of the conventional Schrödinger equation and the $\alpha$-scale. In this case

$$
b=\alpha
$$

and

$$
U_{Q}(\Psi)=\alpha \int_{\mathbf{R}^{3}}|\Psi(x)|^{2}\left(\ln a^{3}|\Psi(x)|^{2}-1\right) d^{3} x .
$$

Let $\mu \in S_{\text {symp }}(\Omega)$. Then

$$
\begin{gathered}
<\mathcal{H}_{Q}>_{\mu}=\frac{1}{2} \operatorname{Tr} \operatorname{cov}^{\mathrm{c}} \mu\left[-\frac{\mathrm{h}^{2}}{2 \mathrm{~m}} \Delta+\mathrm{V}\right] \\
+\alpha \int_{\Omega}\left(\int_{\mathbf{R}^{3}}|\Psi(x)|^{2}\left(\ln a^{3}|(x)|^{2}-1\right) d^{3} x\right) d \mu(\Psi)=\frac{1}{2} \operatorname{Tr} \operatorname{cov}^{\mathrm{c}} \mu \mathbf{H}+\mathrm{o}(1),
\end{gathered}
$$

$\alpha \rightarrow 0$. Here $\mathbf{H}$ is the quantum Hamiltonian. This indirect estimate of $\alpha$ gives us: $|\alpha| \leq 10^{-15} \mathrm{eV}$. Of course, this is only the upper bound and it might be that $\alpha$ should be, in fact, essentially less than $10^{-15} \mathrm{eV}$.

\section{Hamilton function for conventional non- linear Schrödinger equations}

In the conventional nonlinear quantum mechanics, see [13], [15], there are considered equations of the form:

$$
i h \frac{\partial \psi}{\partial t}=\left[-\frac{h^{2}}{2 m} \Delta+V+F\left(|\psi|^{2}\right)\right] \psi
$$


In fact, the Hamilton function for this equation was given already in [13] (but without interpreting the equation (33) as the Hamilton equation in the infinite-dimensional phase space). Following [] we set $U_{Q}(\Psi)=\frac{1}{2} \int_{\mathbf{R}^{3}} d^{3} x\left(\int_{0}^{|\Psi(x)|^{2}} d q F(q)\right)$. Then $U_{Q}^{\prime}(\Psi)=F\left(|\Psi(x)|^{2}\right) \Psi(x)$. We pay attention that $U_{Q}(\Psi)$ is $J$-invariant. To apply our theory, we need at least the $C^{2}$-class for $U_{Q}: \Omega \rightarrow \Omega$, thus at least $C^{1}$-class for $F: \mathbf{R} \rightarrow \mathbf{R}$.

Results of this paper were presented in a series of author's talks on PCSFT - at Steklov Mathematical Institute of Russian Academy of Science (sections of mathematical and theoretical physics as well as the general institute's seminar), at Moscow Institute of Physical Engineering, at University of Mannheim (department of mathematics), at University of Bonn (department of stochastics), at Humboldt University of Berlin (quantum optics laboratory). I would like to thank S. Albeverio, O. Benson, E. Binz, A. Ezhov, A. Slavnov, V. Vladimirov and I. Volovich for hospitality.

\section{REFERENCES}

1. A. Yu. Khrennikov, Phys. A: Math. Gen. 38 (2005) 9051; Found. Phys. Letters 18 (2005) 637.

2. B. Mielnik, Commun. Math. Phys. 31 (1974) 221.

3. R. Haag and U. Bannier, Commun. Math. Phys. 60 (1978) 1.

4. J. Bell and B. Hallet, Philos. Sci. 49 (1982) 355.

5. L. de la Pena and A. M. Cetto, The Quantum Dice: An Introduction to Stochastic Electrodynamics, Kluwer, Dordrecht, 1996; T. H. Boyer, A Brief Survey of Stochastic Electrodynamics, in Foundations of Radiation Theory and Quantum Electrodynamics, A. O. Barut, ed., Plenum, New York, 1980.

6. D. Bohm and B. Hiley, The undivided universe: an ontological interpretation of quantum mechanics, Routledge and Kegan Paul, London, 1993.

7. P. Holland, The quantum theory of motion, Cambridge University press, Cambridge, 1993.

8. E. Nelson, Quantum fluctuation, Princeton Univ. Press, Princeton, 1985.

9. M. Davidson, J.Math. Phys. 20 (1979) 1865; Physica A 96 (1979) 465.

10. P. Morgan, Phys. Lett A 338 (2005) 8; 321 (2004) 216.

11. G. 't Hooft, hep-th/0105105 quant-ph/0212095. 
12. W. M. De Muynck, Foundations of quantum mechanics, an empiricists approach, Kluwer, Dordrecht, 2002.

13. I. Bialynicki-Birula, J. Mycielski, Annals of Physics 100 (1976) 62.

14. A. Shimony, Phys. Rev. A 20 (1979) 394.

C. G. Shull, D. K. Atwood, J. Arthur, and M. A. Horne, Phys. Rev. Letters 44 (1980) 765;

S. Weinberg, Phys. Rev. Letters 62 (1989) 485.

15. H. D. Doebner, Non-linear partial differential operators and quantization, Berlin, Springer-Verlag, 1983.

16. N. Gisin, Hel. Physica Acta 62 (1989) 363.

17. P. A. M. Dirac, The Principles of Quantum Mechanics, Oxford Univ. Press, 1930.

18. J. von Neumann, Mathematical foundations of quantum mechanics, Princeton Univ. Press, Princeton, N.J., 1955.

19. A. Bach, J. Math. Phys. 14 (1981) 125; Phys. Lett. A 73 (1979) 287; J. Math. Phys. 21 (1980) 789.

20. B. J. Hiley, From the Heisenberg Picture to Bohm: a New Perspective on Active Information and its relation to Shannon Information, in Quantum Theory: Reconsideration of Foundations, Ser. Math. Modeling, 2, Växjö Univ. Press, 2002. 